Article

\title{
Investigation of Focused Ion and Electron Beam Platinum Carbon Nano-Tips with Transmission Electron Microscopy for Quantum Tunneling Vacuum Gap Applications
}

\author{
Michael Haub ${ }^{1, * \mathbb{D}}$, Thomas Günther ${ }^{1}$, Martin Bogner ${ }^{1}$ and André Zimmermann ${ }^{1,2}$ (D) \\ 1 Institute for Micro Integration (IFM), University of Stuttgart, 9b Allmandring, 70569 Stuttgart, Germany; \\ thomas.guenther@ifm.uni-stuttgart.de (T.G.); martin.bogner@ifm.uni-stuttgart.de (M.B.); \\ andre.zimmermann@ifm.uni-stuttgart.de (A.Z.) \\ 2 Hahn-Schickard, 9b Allmandring, 70569 Stuttgart, Germany \\ * Correspondence: michael.haub@ifm.uni-stuttgart.de
}

Citation: Haub, M.; Günther, T.; Bogner, M.; Zimmermann, A. Investigation of Focused Ion and Electron Beam Platinum Carbon Nano-Tips with Transmission Electron Microscopy for Quantum Tunneling Vacuum Gap Applications. Appl. Sci. 2021, 11, 11793. https:// doi.org/10.3390/app112411793

Academic Editors: Ştefan Ţǎlu, Sławomir Kulesza and Ilya A. Morozov

Received: 12 November 2021 Accepted: 8 December 2021 Published: 11 December 2021

Publisher's Note: MDPI stays neutral with regard to jurisdictional claims in published maps and institutional affiliations.

Copyright: (c) 2021 by the authors. Licensee MDPI, Basel, Switzerland. This article is an open access article distributed under the terms and conditions of the Creative Commons Attribution (CC BY) license (https:// creativecommons.org/licenses/by/ $4.0 /)$.
Featured Application: The authors integrated platinum carbon (PtC) nano-tips in a tunneling accelerometer. The use of a focused ion beam (FIB) allows flexible and in situ preparation of the tunneling electrodes and other three-dimensional structures in the nanoscale.

Abstract: To realize quantum tunneling applications with movable electrodes, sharp tips with radii down to several tens of nanometers are necessary. The use of a focused ion beam (FIB) and focused electron beam (FEB) with a gas injection system (GIS) allows the integration of geometries in the nanoscale directly into micro and nano systems. However, the implementation of the tunneling effect clearly depends on the material. In this work, a metal-organic precursor is used. The investigation of the prepared tunneling electrodes enables an insight into FIB/FEB parameters for the realization of quantum tunneling applications. For this purpose, a high-resolution transmission electron microscopy (HRTEM) analysis is performed. The results show a dependence of the material nanostructure regarding platinum $(\mathrm{Pt})$ grain size and distribution in an amorphous carbon matrix from the used beam and the FIB currents. The integration of the tips into a polysilicon (PolySi) beam and measuring the current signal by approaching the tips show significant differences in the results. Moreover, the approach of FEB tips shows a non-contact behavior even when the tips are squeezed together. The contact behavior depends on the grain size, proportion of platinum, and the amount of amorphous carbon in the microstructure, especially at the edge area of the tips. This study shows significant differences in the nanostructure between FIB and FEB tips, particularly for the FIB tips: The higher the ion current, the greater the platinum content, the finer the grain size, and the higher the probability of a tunneling current by approaching the tips.

Keywords: tunneling effect; focused ion beam; focused electron beam; platinum carbon; DLC

\section{Introduction}

Focused ion beam-induced deposition (FIBID) and focused electron beam induced deposition (FEBID) enable a wide range of applications at the nanometer scale and show great advantages compared to conventional methods. Due to the high geometrical flexibility, high resolution, and independence from the surface, focused direct irradiation using electron or ion beams allows the fabrication of an almost endless variety of geometrical structures for a wide range of applications. The following are the main applications:

- fabrication of three-dimensional structures in the micro-and nanometer scale [1-4]

- growth of nanowires and nanocontacts [5-8]

- interconnection or repair of circuits [9-11]

- $\quad$ repair of X-ray masks [12] 
More specialized applications are the maskless fabrication of JFETs (junction field effect transistor) [13], the preparation of nanogaps by FEBID [14], or the fabrication of nano-tips facing each other for tunneling applications [15]. Due to the maximum flexibility for post-processing of any nano/microelectromechanical system (NEMS/MEMS) and high patterning resolution, sharp tips with radii in the range of a few nanometers $(\mathrm{nm})$ can be created. To use these tips for quantum tunneling applications, the tips are placed and patterned by a Thermo Fisher Helios NanoLab 600 DualBeam with a GIS and a Trimethyl(methylcyclopentadienyl)platinum(IV) (MeCpPtMe3) metal-organic precursor. Focused beam induced deposition can be divided into three main process steps: First, the precursor gas is introduced and floods the sample's environment. Second, the focused electron/ion beam is activated and scans the sample pixel by pixel in a specific geometric shape and sequence routine. Third, the precursor molecules are split into a non-volatile part, as well as a volatile part. The non-volatile part is deposited on the sample while the volatile part leaves the sample area. Theoretically, only the non-volatile metallic $(\mathrm{Pt})$ part is deposited on the substrate. In fact, a metal-organic material is created, where individual platinum grains are embedded in a high-resistance amorphous carbon (C) matrix. This leads to high contamination of the Pt structure and much higher resistivity compared to bulk Pt. It is known that the conduction mechanism takes place by intrinsic tunneling between the platinum grains [16-20]. Since the tunneling mechanism in these granular metal-organic materials is responsible for the electrical conductivity, the question arises if tunneling through a vacuum gap with the same material is also possible. This opens up a large field of possibilities for the fabrication of novel and miniaturized MEMS and NEMS since the tunneling effect represents a highly sensitive measurement principle. For the use of PtC tips deposited and patterned by the FIB/FEB for quantum tunneling applications with a vacuum gap, the basic suitability and the dependence on the FIB/FEB parameters must be investigated. For this purpose, a series of tips is deposited on a lift-out grid and then investigated using HRTEM to find suitable parameters of FIBID/FEBID to enable quantum tunneling between PtC tips. The evidence for the tunneling effect and the comparison between different $\mathrm{PtC}$ tips is provided by integrating the tips subsequently in a MEMS.

\section{Materials and Methods}

For the investigation by a HRTEM, the PtC nano-tips are deposited and patterned on a typical Omniprobe lift-out grid in a Thermo Fisher Helios NanoLab 600 DualBeam through FIBID and FEBID. The HRTEM analysis is provided by a Thermo Fisher Titan ${ }^{3}$ with a maximum resolution of $0.08 \mathrm{~nm}$. The evidence of the tunneling effect is generated by a movable polysilicon (PolySi) beam structure with an electrostatic actuator fabricated by the PolyMUMPs process of MEMSCAP Inc. Figure 1 summarizes the whole process of the investigation in this study.

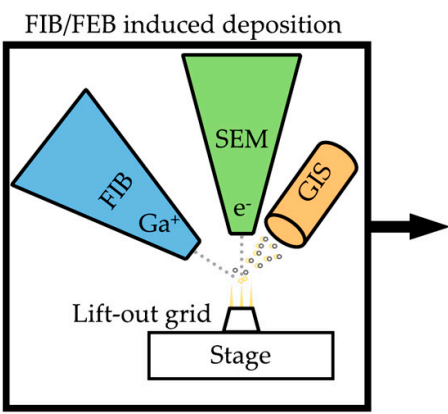

(a)

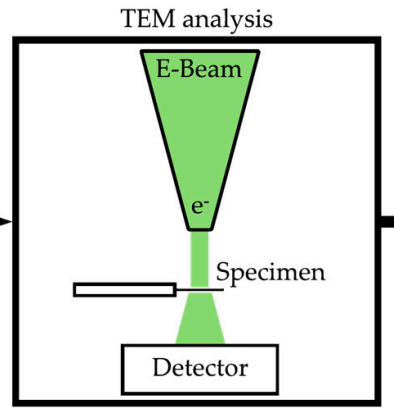

(b)

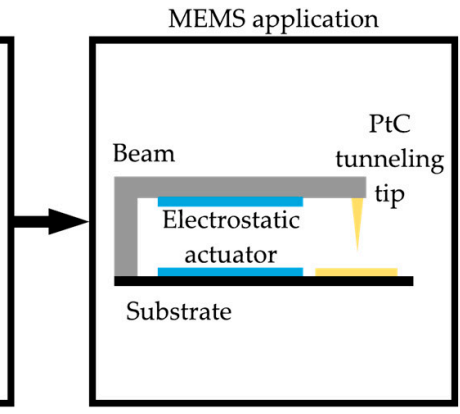

(c)

Figure 1. Process of investigation. (a) Principle of FIB/FEB deposition of PtC nano-tips to an Omniprobe lift-out grid. (b) Principle of HRTEM analysis. (c) MEMS application with integration of a PtC tunneling tip to a movable PolySi beam structure and an electrostatic actuator to approach the tip electrodes facing each other. 


\subsection{Platinum Carbon Composite}

Deposition by FIB/FEB through a Pt precursor is one of the most common variants among a variety of other available material composites with aluminum $(\mathrm{Al})$, gold $(\mathrm{Au})$, tungsten $(\mathrm{W})$, or palladium $(\mathrm{Pd})$, as examples. This investigation is based on the use of a metal-organic precursor material, MeCpPtMe3. In the metal-organic composite, the metallic components are present in an amorphous carbon matrix after deposition. This result has been widely investigated and can be confirmed by previous studies [20-28]. Therefore, compared to bulk $\mathrm{Pt}$, the deposited material can be described as a metal highly contaminated by amorphous carbon impurities. Moreover, Dorp et al. [25] and Utke et al. [26] give a comprehensive overview about processing with focused beam induced deposition (FBID) and the difference in technology between FIBID and FEBID. Labille et al. [21] especially show the bonding structure of the FBID carbon matrix by Raman spectroscopy. The results show a diamond-like amorphous carbon (DLC) based on a sp2 bonding structure corresponding to graphite and sp3 bonding structure corresponding to diamond. The huge amount of high ohmic DLC leads to a high electric material resistance. The study of the resistance of $\mathrm{PtC}$ and the comparison to bulk $\mathrm{Pt}$ has great importance in some publications. Thus, it is deeply investigated and considered from different perspectives how large the resistance is in general and where the dependencies are regarding deposition parameters $[5,20,28-31]$, temperature $[23,28,32]$, technology (FIB/FEB) $[28,30]$ or material thickness $[19,23]$. Tao et al. [31] presented a resistivity dependence on the chosen ion current and measured the lowest resistivity of the deposited nanowires at the highest ion current of $222 \mathrm{pA}$. Previous studies show different values for the resistivity of FIBID and FEBID Pt, but can be adopted as 1-2 orders of magnitude higher for FIBID Pt, and 4-5 orders for FEBID Pt than the resistivity of bulk Pt [19,23,28,31].

The tunneling effect is primarily used in a scanning tunneling microscope (STM) for commercial purposes. Therefore, highly conductive tips are used, and contamination or oxidation is prevented by imaging under vacuum conditions. The first requirement for a high-resolution STM analysis is a pure metal tip with low resistance. In the configuration of a well conductive tip and probe, tunneling currents of up to $100 \mathrm{nA}$ can be expected. Since high ohmic parts of the tunneling tip prevent electron tunneling from one electrode to another, the nanostructure of the $\mathrm{PtC}$ composite, particularly its edge area, needs to be investigated to determine the suitability of the tunneling mechanism. However, the conduction mechanism of metal-organic or granular materials is based on intergrain tunneling between the metallic components [16-20]. Schwalb et al. [33] show the realization of a strain sensor by bending a $\mathrm{PtC}$ nanowire and, therefore, a great change in the material inherent resistance through the exponential dependence of the distance between the metallic grains and the tunneling current. This leads to a resistivity dependence on the size and distance of the metallic components to each other. For a tunneling application through a vacuum gap, this is a significant aspect because if the Pt grains are located directly at the edge of the tip, a tunneling current can occur. This is one of the main aspects of this work, as the application of $\mathrm{PtC}$ tips allows tunneling application through a vacuum gap by suitable location and distribution of Pt grains. Examination by HRTEM represents the most considerable analysis for this purpose since the microstructure of the tip can be imaged on the nano- and picometer scale. Due to the high flexibility of the FIB, the integration of sharp tips enables a wide range of tunneling applications for sensors based on movable parts such as accelerometers or pressure sensors. The high sensitivity of the quantum tunneling effect allows a high level of miniaturization in those sensors because of very low deflection along with great changes in the device signal.

\subsection{Specimen Preparation}

The preparation of the nano-tips for the HRTEM analysis is comparable to those of TEM (transmission electron microscope) lamellas which is state of the art for investigating material compositions. The basic principle of the TEM is to irradiate the sample with a high-energy electron beam. A detector on the backside intercepts the electron beam and 
allows imaging of the atomic structure of the material components. To achieve a high resolution of the nanostructure, the material thickness must not exceed a few $10 \mathrm{~nm}$. Since such dimensions are possible with the FEBID and FIBID, this is the standard technology for TEM specimen fabrication. In this study, the differences in the tips depending on the ionic current used are elaborated. The FEB tips are deposited by a $340 \mathrm{pA}$ electron beam current with an acceleration voltage of $5 \mathrm{kV}$. For the deposition of the FIB tips, currents of $9 \mathrm{pA}, 46 \mathrm{pA}, 90 \mathrm{pA}$, and $260 \mathrm{pA}$ are set in addition to the accelerating voltage of $30 \mathrm{kV}$. The process of preparation of the PtC nano-tips is shown in Figure 2.
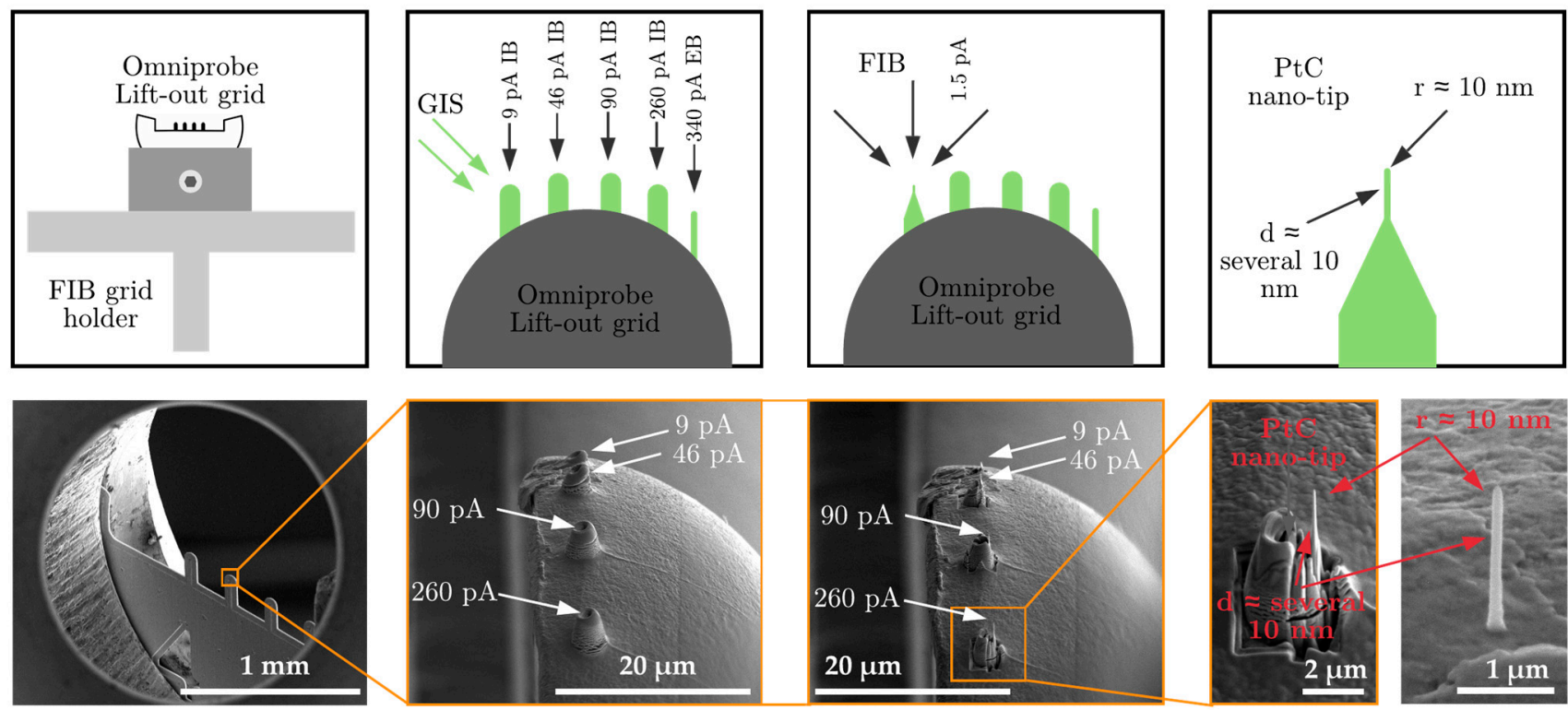

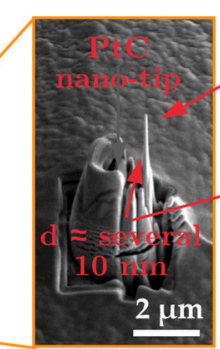

(d)

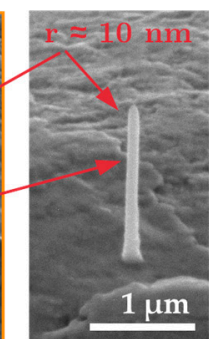

(e)

Figure 2. Process of preparation of the PtC nano-tips for the HRTEM analysis with (a) the Omniprobe lift-out grid, (b) the FIB and GIS deposition of PtC bumps, (c) the principle of patterning of the bumps to sharp nano-tips by a low FIB current of $1.5 \mathrm{pA}$ with (d) radii $\mathrm{r}$ approximately of $10 \mathrm{~nm}$ and thickness $\mathrm{d}$ of a few $10 \mathrm{~nm}$ and (e) the deposited FEB nano-tip without additional patterning. Above the principle of the process step and below the SEM image are shown.

To fabricate the $\mathrm{PtC}$ nano-tips, a standard Omniprobe lift-out $\mathrm{Cu}$ (copper) grid is used and placed on a FIB holder (Figure 2a). This is necessary to ensure compatibility for the TEM. Usually, a lift-out grid is used to place a previously fabricated lamella by the FIB on it. In the current study, the whole preparation process is provided directly to the grid. After cleaning the top of the grid by a large-scale cleaning process with the FIB, the PtC bumps for the fabrication of the nano-tips are deposited. Figure $2 \mathrm{~b}$ shows the principle and result of the deposition of five bumps with ion currents of $9 \mathrm{pA}, 46 \mathrm{pA}, 90 \mathrm{pA}$, and $260 \mathrm{pA}$, as well as an electron current of $340 \mathrm{pA}$. During the deposition, the precursor gas is induced by the GIS needle. Unlike the FEBID, it is not possible to directly fabricate sharp tips of a few $10 \mathrm{~nm}$ in thickness using the FIB deposition. Therefore, the bumps are patterned subsequently by the FIB with a minimal and sensitive ion current of $1.5 \mathrm{pA}$ (Figure 2c). This step is the most challenging part to provide suitable structures in the nanoscale for the HRTEM analysis. Since the imaging with the ion beam comes along with a destructive removal of the specimen, several short steps with a targeted patterning of the bumps from different angles and directions are necessary. Figure $2 \mathrm{~d}$,e shows the result exemplarily for the 260 pA FIB tip in the side view and the 340 pA tip deposited by the FEB. As the tip becomes thinner, as more information can be found by the HRTEM about the material's atomic structure. 


\subsection{Tip Integration to MEMS}

This work aims to investigate the possibility of using PtC tips in quantum tunneling vacuum gap applications. Therefore, the results and findings of the HRTEM analysis will be validated in a MEMS consisting of a PolySi beam and an electrostatic actuator. After deposition and patterning of the nano-tip to the beam, the tunneling effect can be proved by measuring the tunneling current between two PtC electrodes at distances of some nm. For a deeper insight into this subject, the authors published their work in another survey [15]. The process of tip integration to the MEMS is shown in Figure 3.

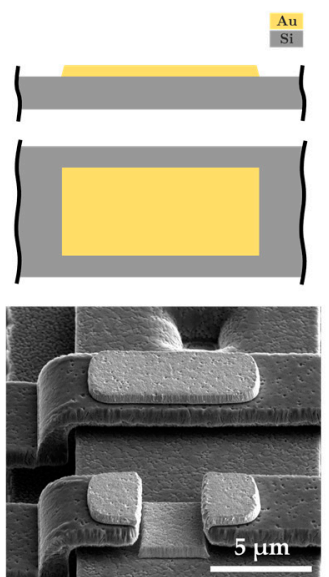

(a)
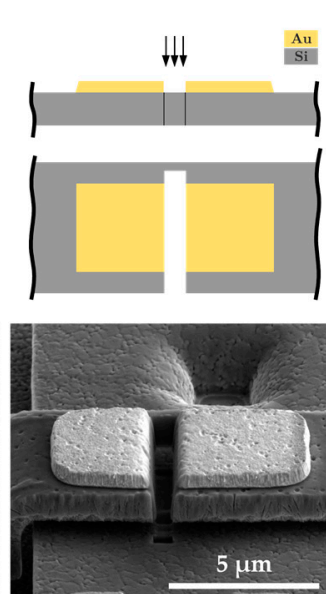

(b)
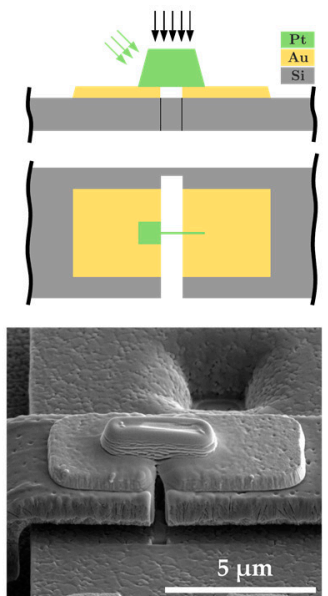

(c)
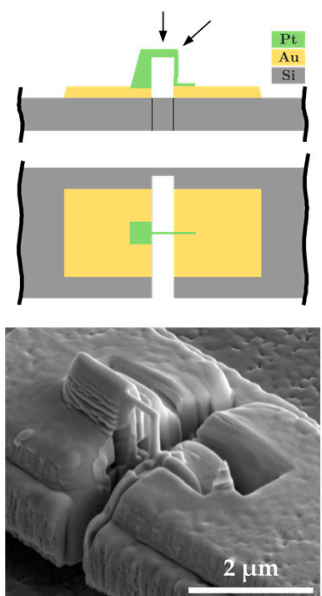

(d)
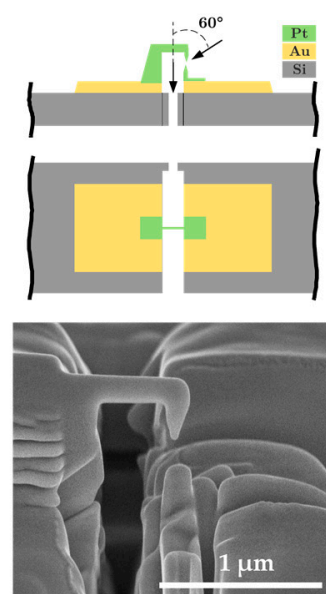

(e)

Figure 3. Process of tip integration to the MEMS with (a) the initial state of the PolySi beam, (b) the basic patterning of the beam to obtain a fixed and a movable part, (c) the deposition of a $\mathrm{PtC}$ pad, (d) patterning of the PtC pad and (e) the final PtC nano-tips/electrodes by dividing the nanobeam by the FIB.

Figure 3a shows the initial state of the PolySi beam with a thin gold $(\mathrm{Au})$ layer for a high conductivity of the structure. The fabrication of the MEMS is carried out by the foundry service PolyMUMPs from MEMSCAP Inc. and subsequent micro structuring of the tunneling electrodes with a focused ion beam. PolyMUMPs is a surface micromachining process with three polysilicon layers, two sacrificial layers, and a metal layer. The minimum structure size for PolyMUMPs is $2 \mu \mathrm{m}$. The standardized process procedure with strict adherence to the design rules ensures low manufacturing times and costs. Since the application of this tunneling device proves a vacuum gap tunneling mechanism by varying the tip distance, the PolySi beam must be divided into movable and fixed parts. Therefore, the PolySi beam is patterned by the FIB in the first process step illustrated by Figure 3b. As shown in the preparation of the tips for the HRTEM analysis, it is necessary to fabricate the nano-tips in several steps. After deposition of a PtC pad by the FIB or the FEB and the GIS (Figure 3c), the pad is milled subsequently by the ion beam to obtain a thin vertical nanobeam (Figure $3 \mathrm{~d}$ ) and a final cut with a small ion current of $1.5 \mathrm{pA}$ to provide two opposing tunneling tips (Figure 3e). The smallest size of the initial PtC pad depends on the technology and chosen beam current. The FEBID allows a much finer deposited structure than the FIBID. Moreover, the same can be seen on different ion currents. As lower the ion current is chosen, as higher the resolution of the deposited pad. The initial pad in Figure $3 \mathrm{c}$ is based on the FIBID with a $260 \mathrm{pA}$ current. Due to attractive forces between the tips [15], the tips' radii are patterned by the FIB as sharply as possible. To measure and verify the tunneling current between the PtC tips, the MEMS is connected to a two-channel Source Measuring Unit (SMU) Tektronix Keithley 2614b, as shown in Figure 4a. The MEMS-Chip is bonded by a Wire Bonder G5 Single (F\&K Delvotec) to a printed circuit board (PCB) (Figure $4 \mathrm{~b}$ ). One channel operates the electrostatic actuator to approach the tip of the movable part towards the fixed tip below. The second channel is loaded by the tunneling bias voltage in the range of $100 \mathrm{mV}$ to $1 \mathrm{~V}$ and measures the 
occurring tunneling current at final tip distances of a few nm. Figure 4c shows the PolySi beam structure with the actuator in top view, and Figure $4 \mathrm{~d}$ depicts the final PtC tip in side view. Although this application enables the measurement of external forces to the MEMS (in the case of an accelerometer or pressure sensor), in this study, the static approach of the tips is only provided to prove the ability of quantum tunneling between organic-metal materials and their dependence on the FIB/FEB fabrication parameters. The approach of the tips is performed by several small voltage steps $(1-5 \mathrm{mV})$ using the electrostatic actuator. The measuring point for every voltage step on channel 1 and the tunneling current on channel 2 is saved by the SMU to a log file. Afterwards, the log file is evaluated to show the course of the current measurement between the tunneling electrodes/tips. Once the current rises, the slope shows an exponential character for the tunneling current or a step in terms of an ohmic contact indicating a crash of the electrodes. All measurements are carried out in the dual-beam chamber under high vacuum conditions to prevent contamination of the tunneling tips and keep the environmental conditions as constant as possible. For the sensor-technical implementation of the susceptible tunneling effect, sources of disturbances must be avoided as far as possible. The connection from the SMU to the MEMS chip is made via a cable lead-through on the vacuum chamber.

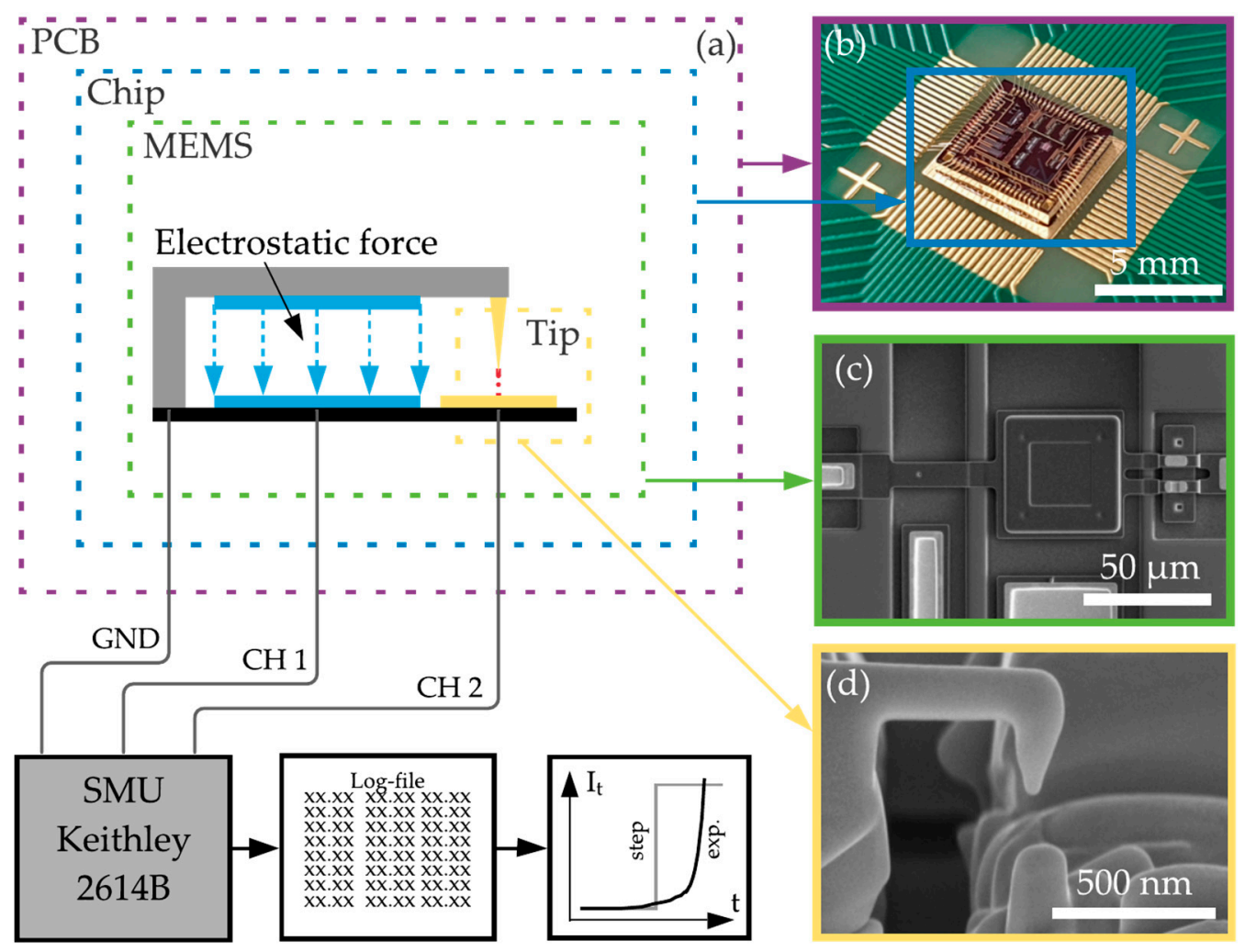

Figure 4. Principle of wiring (a) and the bonded chip (b) for the tunneling application in a MEMS (c) with a two-channel Keithley SMU 2614B. Ground (GND) is connected to the PolySi beam, channel 1 $(\mathrm{CH} 1)$ to the substrate pad of the electrostatic actuator, and channel $2(\mathrm{CH} 2)$ to the counter electrode of the tunneling tip (d).

\section{Results}

The following results show the findings from the HRTEM analysis comparing the measurements and elaborating the differences on each PtC nano-tip for ion beam currents of $9 \mathrm{pA}, 46 \mathrm{pA}, 90 \mathrm{pA}$, and $260 \mathrm{pA}$ as well as an electron beam current of $340 \mathrm{pA}$. Testing in the FIB deposition shows an increased scattering of the deposited material when higher ion currents are applied, as well as an inversion between the etching and depositing processes, leading to a material removal rather than a deposition. For this reason, a maximum ion 
current of $260 \mathrm{pA}$ is chosen. The findings are subsequently proven by implementing the tips to a MEMS to show the tunneling current or otherwise the crash of the tips marked by an instant ohmic contact.

\subsection{Transmission Electron Microscopy Analysis}

The results of the HRTEM analysis are described by several steps beginning with an overview of the tips to evaluate the differences of the nanostructure, subsequently showing the grain size in a magnified image for the center and edge area of the tip, and finally investigating the lattice spacings of the atomic structure by performing the fast Fourier transformation (FFT) of the structure. The lattice constant of face-centered cubic (FCC) Pt (space group $\mathrm{Fm}-3 \mathrm{~m}$ ) is $3.9231 \AA$ at room temperature according to the PDF $4+$ card \# 00-004-0802 [34]. The interplanar spacings for Pt of the 111, 200, 220, and 311 planes with high intensity can be calculated for the FCC crystal system by

$$
d_{h k l}=\frac{\mathrm{a}}{\sqrt{h^{2}+k^{2}+l^{2}}}
$$

where the Miller indices are $h, k, l$, the lattice constant is a, and the interplanar spacing is $\mathrm{d}$.

To show the difference in the nanostructure of the tips, the overview images of the 9 pA, 46 pA, 90 pA, and 260 pA FIB, as well as the 340 pA FEB tips, are shown in Figure 5a-e. From the $9 \mathrm{pA}$ to the $260 \mathrm{pA}$, a decrease of the grain size from a blurred to a more granular structure becomes apparent regarding the whole tip shown. Concerning this, Figure $5 \mathrm{f}-\mathrm{j}$ illustrates a magnified view of the end of the tip to perform the FFTs in Figure 5k-o. In the images shown, the differences tend to be distinguishable in terms of grain size. However, the FFT gives a much clearer insight into the differences with respect to the diffraction rings of the nanostructure. An increase in intensity, particularly of the outer rings corresponding to the 220 and 311 planes, can be seen. According to Table 1, the interplanar spacings of these planes are 1.3873 and $1.1826 \AA$ and thus just above the resolution limit. The 111 and 200 planes are detectable in all FFTs due to the much larger interplanar spacing. This leads to a decrease in intensity from the center to the outside of the diffraction pattern. Depending on the grain size in the nanostructure, the planes with smaller spacing can be detected or not. Thus, a direct comparison of the different tips shows an increase in the number of smaller grains in which the 220 and 311 planes become visible. Specifically, a slight increase in intensity can be seen from the 9 pA to the $46 \mathrm{pA}$ and $90 \mathrm{pA}$ tips. In contrast, a significant jump in intensity can be seen at the 260 pA tip and the 340 pA FEB tip. As a result, there is a correlation between the ion current or the use of the FEB and the intensity of the diffraction rings. According to the visual inspection of the images, the FFTs confirm the observation of a decreasing grain size when the ion current is increased or the FEB is used.

Table 1. Interplanar spacings of the FCC Pt crystal structure with a lattice constant of $3.9231 \AA$ according to the PDF $4+$ card \# 00-004-0802 [34].

\begin{tabular}{cc}
\hline Plane & $\mathrm{d}[\AA ̊$ \\
\hline 111 & 2.2650 \\
200 & 1.9616 \\
220 & 1.3873 \\
311 & 1.1826 \\
\hline
\end{tabular}

In the following, only the $9 \mathrm{pA}$ and $260 \mathrm{pA}$, as well as the $340 \mathrm{pA}$ FEB tips, are considered because of the specific differences. A different view of the tips is provided by the binary images of the HRTEM recordings. Figure 6 shows the tips after blurring through a $10 \times 10$ pixel matrix and binarization at a threshold of gray values $(0=$ black to $255=$ white) of 80 . Blurring highlights larger grains and reduces the "noise" of very small contours in the image. Binarization enhances the contrast between light and dark areas in the image. In addition, the binary image $(80 \%)$ is merged to the original transparent 
image (20\%) to visualize the actual edge of the tip while the blue markers highlight the particularly large grains in Figure $6 \mathrm{~b}$ and create a comparison to the grain size in Figure $6 \mathrm{a}, \mathrm{c}$. The red markings show the distribution of grains in the direct edge region of the top edge since only these grains contribute to quantum mechanical tunneling through a vacuum gap. The FEB tip in Figure 6a shows no grains in the upper edge area in the binary image. Here, a clear difference in the number of grains can be seen by a comparison of the binary images. The $260 \mathrm{pA}$ tip shows a much more homogeneous distribution of grains in the edge region, while only a few grains are visible in the 9 pA tip. Of course, when the threshold is increased, more grains are visible. However, the binary images' relative representation is only intended to allow a direct comparison of the tips and their differences. Furthermore, there is a clear difference in the spacing and distribution of the grains throughout the entire nanostructure. The FEB tip in Figure 6a shows a very homogeneous distribution of the grains all over the nanostructure. For the FIB tips, the number of grains in Figure $6 \mathrm{c}$ is much larger; therefore, the spacings between the grain are much lower than in Figure $6 \mathrm{~b}$.
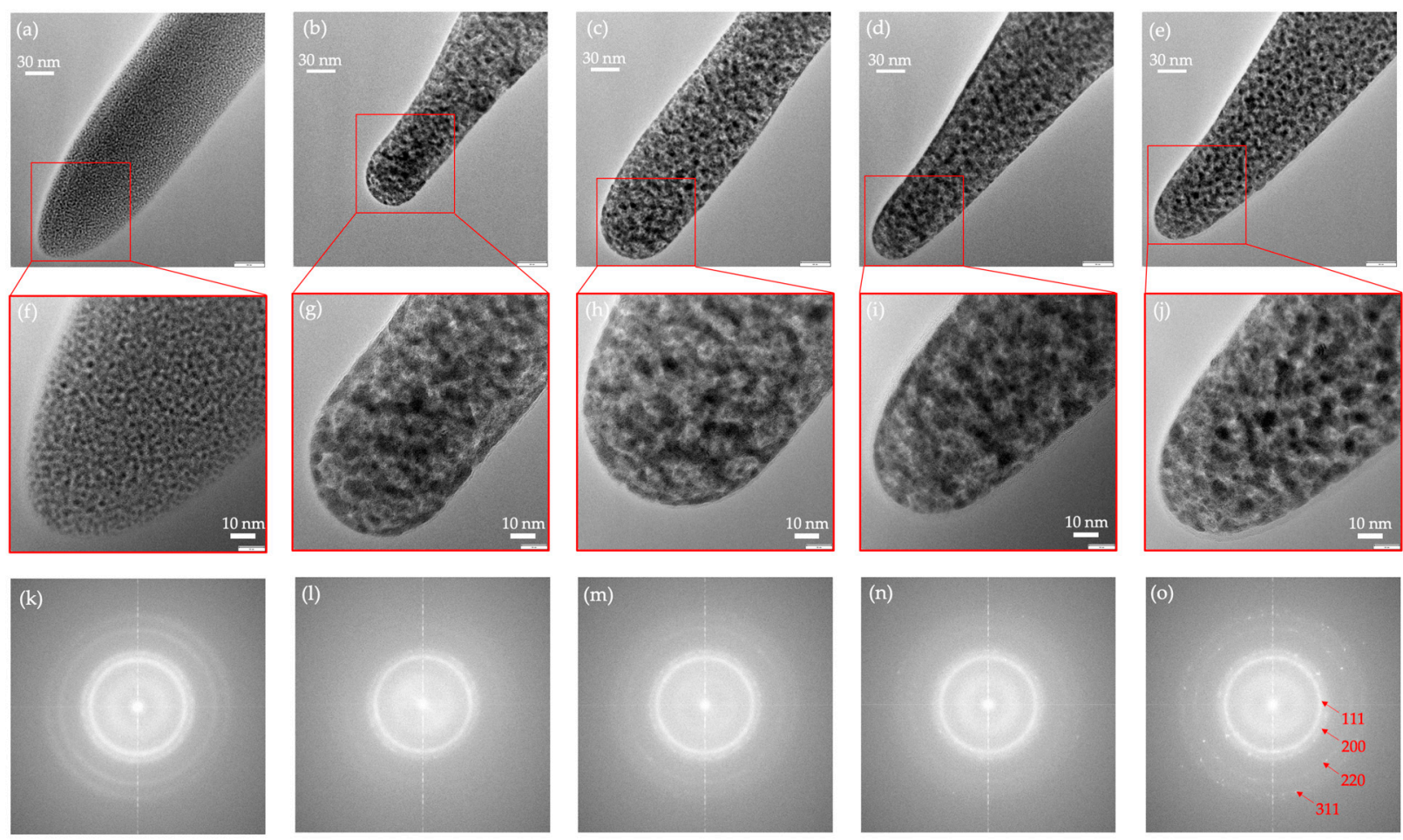

Figure 5. HRTEM analysis overview images of the PtC nano-tips (a) 340 pA EB (b) 9 pA IB, (c) 46 pA IB, (d) 90 pA IB and (e) 260 pA IB. (f-j) the magnified images of the tips for the FFTs $(\mathbf{k}-\mathbf{o})$.

In order to deepen previous investigations, the tips from the $9 \mathrm{pA}$ and $260 \mathrm{pA}$ ion current and the 340 pA FEB tip are again significantly enlarged. Figure $7 a-c$ shows the HRTEM images of the tip ends. The left side in Figure 7a-c shows the attempt to capture the grains using the brightness differences. Characteristic for the HRTEM analysis are different gray values in the image depending on the intensity of the penetrating electron beam. That is, the thicker the grains, the darker they appear. In the comparison of the three top images of Figure 7, it is thus repeatedly visible in detail that the grain size of the tips differs significantly. The grains from the FEB tip in Figure 7a and the 260 pA FIB tip in Figure 7c are clearly smaller and more densely distributed than the 9 pA FIB tip in Figure $7 \mathrm{~b}$. Due to a more homogeneous distribution of the grains, it is possible to conclude that there is a much lower probability of defects in the edge area of the tip, as already discussed in Figure 6. Otherwise, it can be seen that the amorphous carbon areas in the FEB tip take up more space than in the FIB tips. This, in turn, leads to a decrease of Pt grains in the edge area. The right half of Figure $7 \mathrm{a}-\mathrm{c}$ and the subsequently selected areas electron 
diffraction (SAED) and their FFTs deepen the investigation from Figure 5. Figure 7d first shows a SAED of the 340 pA FEB tip and clearly visible interplanar planes in the FFT (Figure 7e) corresponding to the 111, 200, 220, and 311 planes. The 9 pA FIB (Figure 7f) tip shows only the interplanar planes of the 111 and 200 planes. Additionally, the FFT from Figure $7 \mathrm{~g}$ confirms this by the intensity of the diffraction rings and the absence of the outer rings corresponding to the 220 and 311 planes, respectively. Figure 7h shows the FFT and Figure $7 \mathrm{i}-\mathrm{k}$ the SAEDs of the $260 \mathrm{pA}$ tip with much smaller grains. The difference in the intensity of the diffraction rings and spots is obvious. Moreover, with similar intensity of the 111 and 200 levels, visibility of the 220 and 311 levels is given. Since the 311 planes with an interplanar spacing of $1.1826 \AA$ is already close to the resolution limit of the HRTEM of $0.8 \AA$, this grating can only be detected by marking the diffraction spot and the inverse FFT. On both the 260 pA FIB and the 340 pA FEB tip with their common feature of small grains, the small interplanar planes are visible through the FFTs and the SAEDs. Thus, this leads to a correlation between the grain size and the intensity of the diffraction rings. Since the thickness of the tips is larger than the grains, amorphous regions of the DLC are overlaid by the lattice structures of the $\mathrm{Pt}$, as well as several $\mathrm{Pt}$ grains by one another. Amorphous regions are always visible between the grains, as shown by the yellow marking in Figure 7d. However, it is difficult to study these regions and make detailed conclusions.
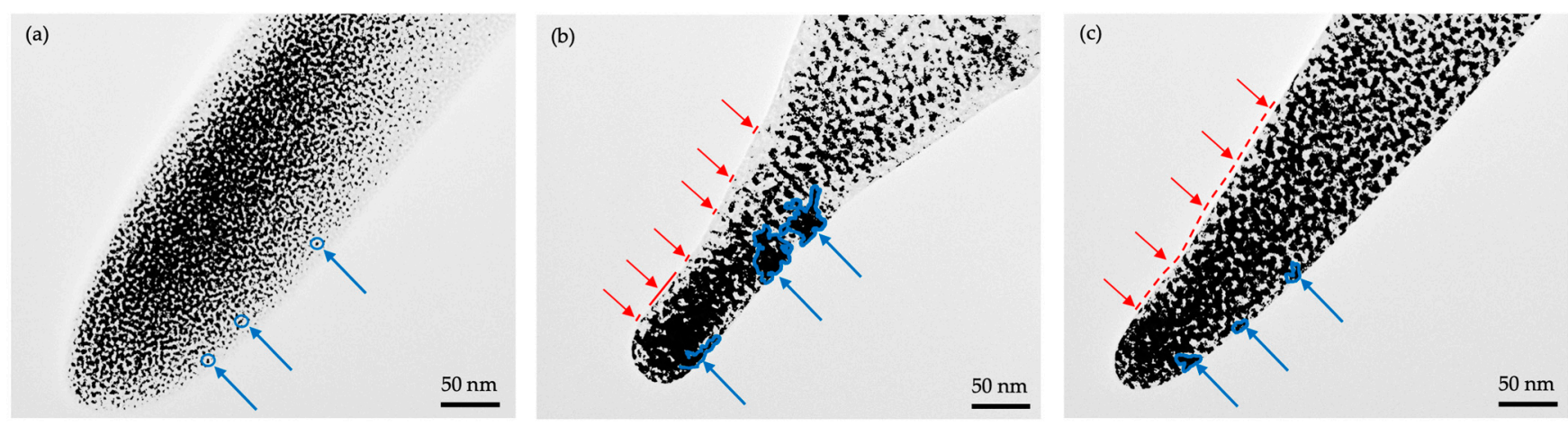

Figure 6. Binary images of (a) the 340 pA FEB, (b) the 9 pA, and (c) the 260 pA FIB tip with the merged transparent original images to show the edge of the tips. Red markings show the grain distribution in the edge area, and blue markings the comparison of the grain size.

All previous data on the grains refer to the accumulation of Pt crystallites. The clusters appear darker in the HRTEM images than areas simply transmitted by the electron beam. For a deeper insight into the Pt nanostructure it is worthwhile to consider the individual crystallites and their size in addition. Figure 8 shows the measurement of the max. length of each of twenty individual crystallites from the 340 pA FEB tip (mean $=2.54 \pm 0.57 \mathrm{~nm}$ ), and the $9 \mathrm{pA}$ (mean $=4.13 \pm 0.88 \mathrm{~nm}$ ), as well as the $260 \mathrm{pA}($ mean $=3.99 \pm 1.31 \mathrm{~nm}$ ) FIB tip. The size of the crystallites by FEBID can be clearly distinguished from the crystallites by FIBID by around $1.5 \mathrm{~nm}$. Hence, there is no markable difference in the crystallite size for the two FIBID tips, the main difference can be determined in the accumulation of several crystallites to smaller and bigger Pt grains, as shown in Figure 7. 

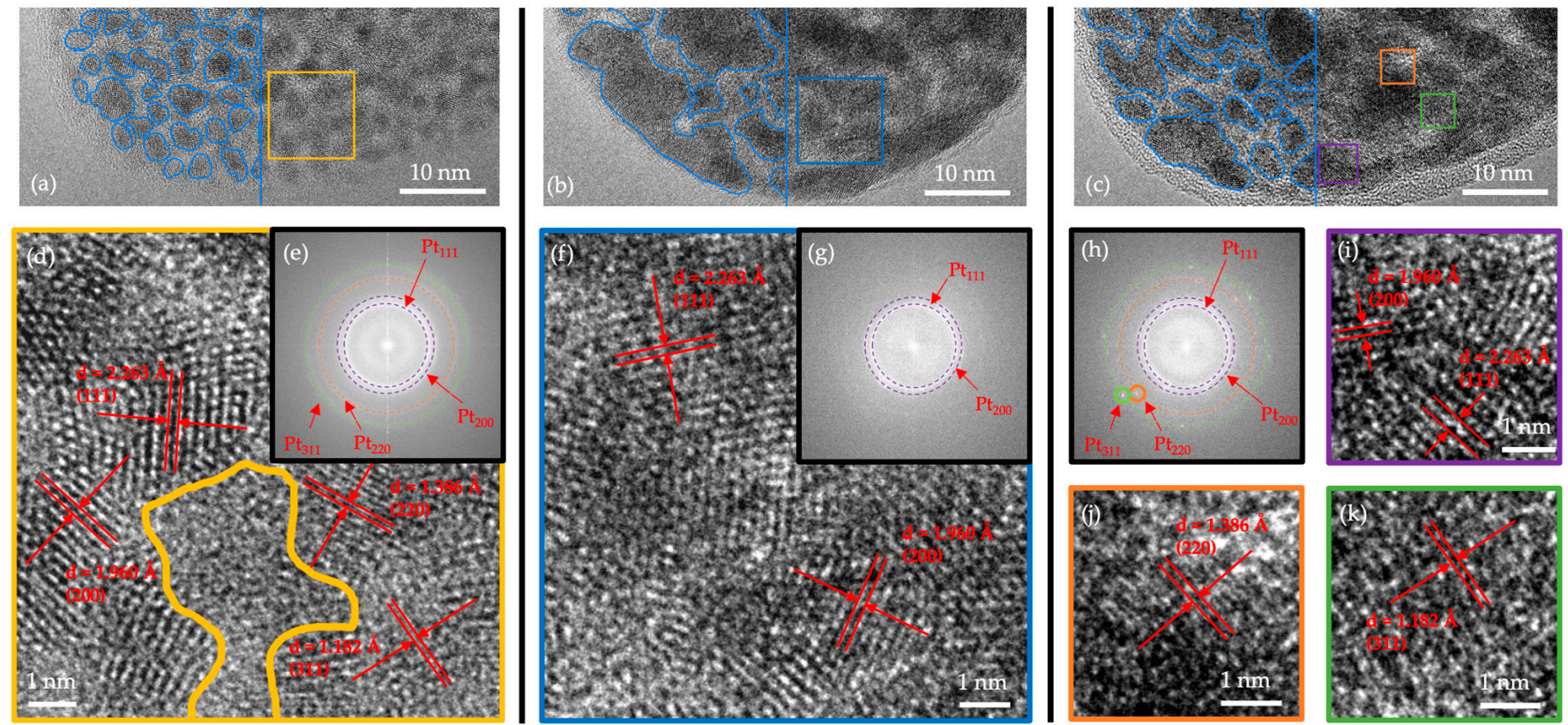

Figure 7. Images of (a) the 340 pA FEB, (b) 9 pA and (c) the 260 pA FIB tip, $(\mathbf{d}, \mathbf{f}, \mathbf{i}, \mathbf{j})$ and $(\mathbf{k})$ the SAEDs and (e,g) and (h) its FFTs.

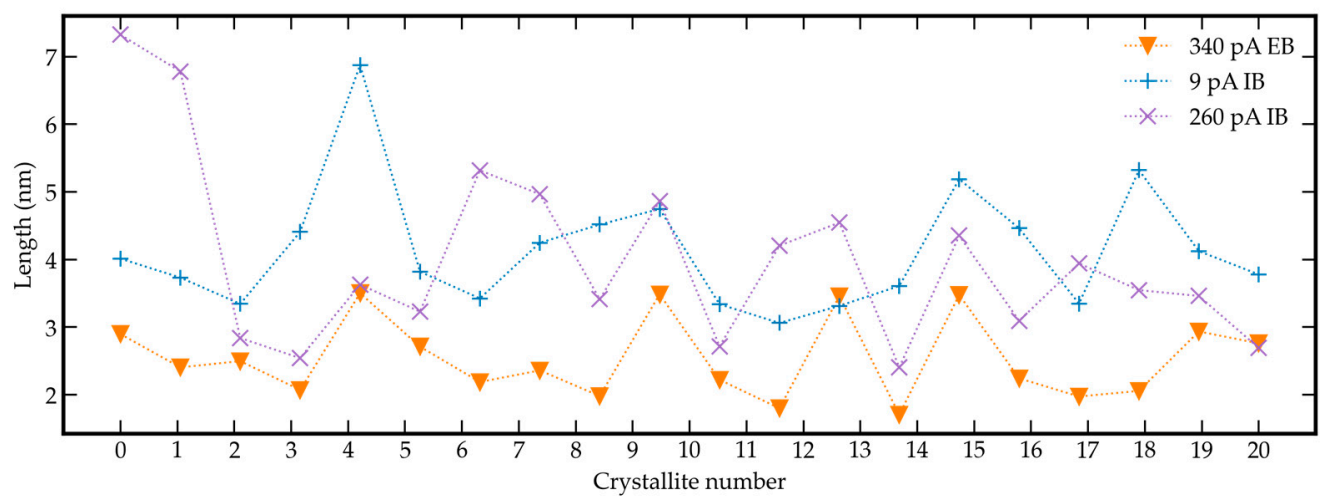

Figure 8. Measurements of twenty individual Pt crystallite of the 340 pA FEB, 9 pA FIB and 260 pA FIB tips.

\subsection{Tunneling Verification}

In accordance with the findings from the HRTEM analysis, the $340 \mathrm{pA}$ FEB and the $9 \mathrm{pA}$ and $260 \mathrm{pA}$ FIB tips are tested for their suitability for the quantum mechanical tunneling mechanism across a vacuum gap by integration into a MEMS. Figure 9 shows the measurements when the tips approach distances of a few $\mathrm{nm}$ at which the tunneling effect occurs and the subsequent removal of the tips. Since measurements with the FEB tip show no increasing current, either as contact or tunneling behavior, the presentation of the measured results is omitted. It is suggested that the huge amount of DLC, especially in the edge area, leads to a high ohmic contact; therefore, no current flows between the electrodes. In the case of the PtC tips, the tunneling effect only occurs when two Pt grains face each other at the tunneling distance of a few $\mathrm{nm}$. If two Pt grains meet each other, an exponentially increasing current curve occurs when the distance is shortened linearly. Thus, the electrostatic behavior is quadratic regarding the deflection by increasing the actuator voltage. However, the deflection range concerning the tunneling distance is so small that it can be assumed to be quasi-linear. When a Pt grain or an amorphous DLC region meets another DLC region, either no current flows or only when the tips get into ohmic contact. In the latter case, this is visible by a jump in the current waveform. According to the excitation principle, the actuator voltage (green line) is increased until the tunneling electrodes are 
only a few nm apart. The voltage values from Figure $9 \mathrm{a}, \mathrm{b}$ depend on the initial distances of the tips and therefore defer from each other. The orange line in Figure $9 a, b$ marks the current threshold at $100 \mathrm{pA}$ for the measurement algorithm to detect the occurrence of the tunneling or contact current and to retreat the electrode. For the measurements, a tunneling voltage of $1 \mathrm{~V}$ is applied to each of the electrodes. Figure 9 shows reproducible curves of the current over the measurement time with repeated approaches and removal of the tips. The offset of the measurements is slightly below the $0 \mathrm{~A}$ mark in each case due to leakage currents through the wiring, the measurement system, and the conductive tracks on the PCB and MEMS chip. The measurement algorithm causes the voltage to increase in 1 to $5 \mathrm{mV}$ steps on the electrostatic actuator until the current threshold is reached. The voltage is then lowered to move the tips away from each other and then approached again. Figure 9 a shows the measurement when the 9 pA tip is approached. In most cases, the current waveform can be seen as a drop in the device signal. This indicates an instant ohmic contact without electron tunneling. Accordingly, an electrical connection is established only by direct contact of the tips corresponding to the conduction mechanism in metal-organic materials. After a sudden increase of the current by contact of the electrodes and subsequent decrease of the actuator voltage, the electrodes adhere to each other by the surface forces until the counteracting force of the PolySi beam overcomes them. This moment can be seen by the abrupt drop in the current waveform back to $0 \mathrm{~A}$. Additionally, the characteristic gradually decreasing current is marked by the red arrows caused by a decreasing attractive force due to the tips pulling apart. Figure $9 \mathrm{~b}$, on the other hand, shows the characteristic measurement with a 260 pA FIB tip. With these tips, an exponentially increasing current waveform is apparent when approaching, and an exponentially decreasing current waveform is apparent when moving away the electrodes. With respect to the slope, height, and decrease of the current, the high sensitivity of the tunneling effect leads to slightly different curves, especially the actuator voltage, to overcome the current threshold. The points where the tunneling current appear are marked by the blue dots and lines. It can be seen that there is no significant difference between the single approaches regarding the actuator voltage operating point. Due to the high intrinsic resistance, tunneling currents of a few $\mathrm{nA}$ to a few $10 \mathrm{nA}$ cannot be achieved with PtC tips, as is common when using metallically pure electrodes in scanning tunneling microscopes. As the measurements from Figure 9 show, the currents at a tunneling voltage of $1 \mathrm{~V}$ are in the range of 100-200 pA, even with an ohmic contact. 

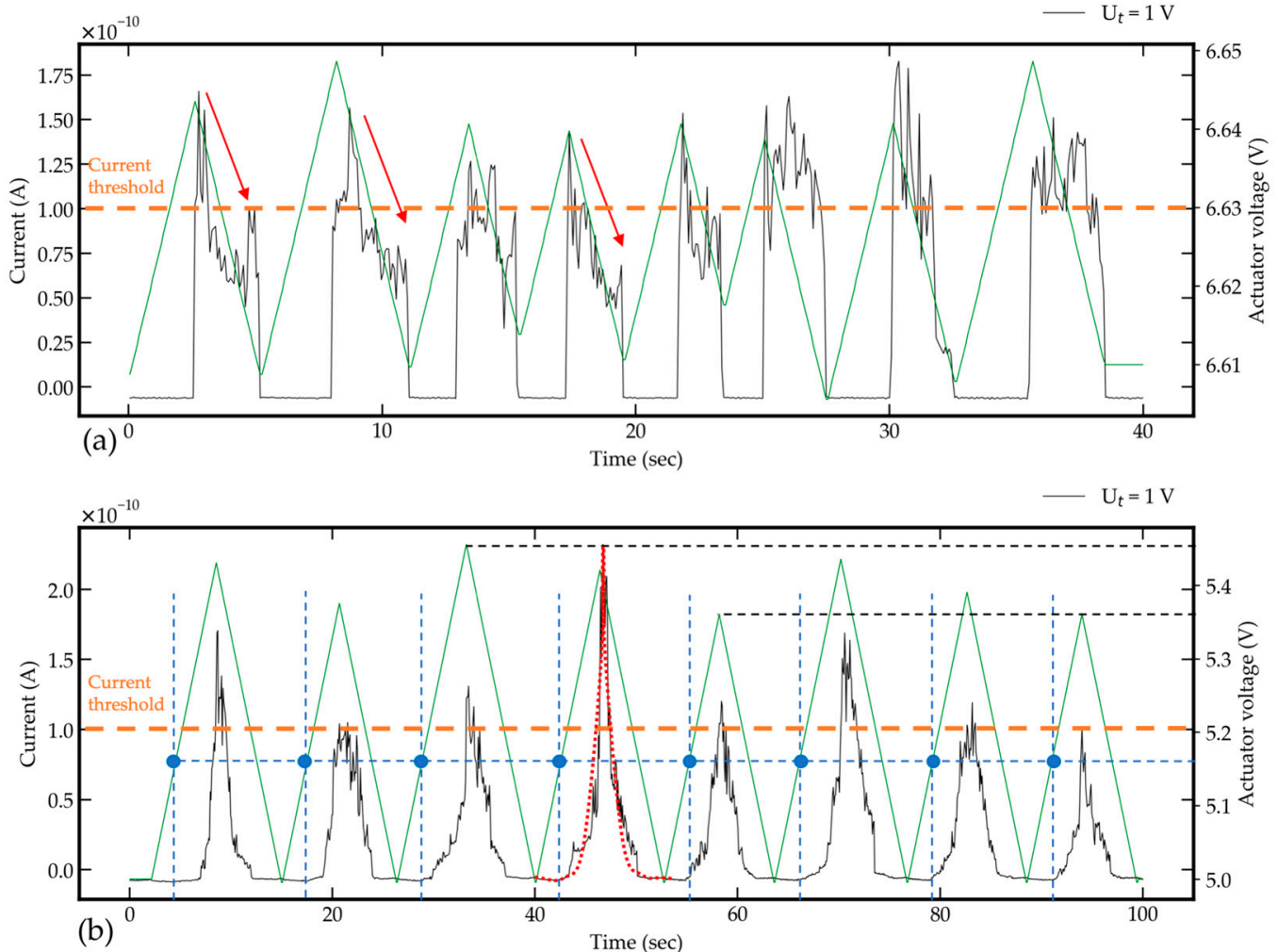

Figure 9. Measurements of tunneling application in a MEMS with integrated tunneling tips with (a) the measurement of the $9 \mathrm{pA}$ and (b) of the $260 \mathrm{pA}$ FIB tip. The excitation (green) is shown by the waveform of each actuator voltage range, differing from each other due to the initial distances of the tips. $\mathrm{U}_{\mathrm{t}}=$ tunneling bias voltage.

\section{Discussion}

The use of PtC tips for applications based on the quantum mechanical tunneling effect depends on the distribution, size, and the number of Pt grains in the nanostructure. These Pt grains are embedded in a DLC matrix. The intrinsic conduction mechanism is based on electron tunneling between the individual $\mathrm{Pt}$ grains [17]. The more densely the $\mathrm{Pt}$ grains are distributed, the lower the resistivity of the material [33]. For tunneling between two PtC tips through a vacuum gap, the same conditions apply except for the additional distance through the vacuum gap. Thus, if the Pt grains are not directly placed at the edge of the tip but are surrounded by the high resistivity DLC, the tunneling mechanism does not appear at all shown in Figure 9a. The tunneling mechanism is supported by increasing the tunneling bias voltage. However, attractive forces play a crucial role when using a moving tip over a fixed tip, as shown by Haub et al. [15]. Since this depends particularly on the tunneling voltage, only small tunneling voltages can be used for this application. In addition, electron tunneling can only be realized with small grain spacings across the vacuum gap. The HRTEM analysis shows a difference in the PtC nanostructure depending on the FEBID and FIBID as well as used currents (Figure 5). Produced tips by the FEBID show a homogeneous distribution of Pt grains, but an increased amount of DLC. This leads to an increased occurrence of DLC regions in the edge region, which prevents electron tunneling (Figure 7a). The FIBID tips show a difference in nanostructure depending on the ion current used. The $9 \mathrm{pA}$ tips have larger Pt grains and thus lower density in the distribution, resulting in larger inter-grain spacing (Figure 7b). The $260 \mathrm{pA}$ tips have much smaller grains, greater density, and smaller spacing between Pt grains (Figure 7c). When binarizing the HRTEM images, it is noticeable that there is a more homogeneous occurrence of $\mathrm{Pt}$ grains in the edge region for the $260 \mathrm{pA}$ tips compared to the $9 \mathrm{pA}$ tips. The measurement of individual crystallites shows on average $1.5 \mathrm{~nm}$ longer lengths for the FIBID tips compared to the FEBID tips. However, there is only a 
small difference between the FIBID tips. Therefore, the results of De Teresa et al. [23] on the independence of the crystallite size from electron or ion beam cannot be confirmed. When the tips are approached in the MEMS application with small tunneling bias voltages, the measurements show a non-contact behavior for the FEBID tips, a drop in the current course for the $9 \mathrm{pA}$ FIBID tip, and an exponentially increasing current course corresponding to the expected quantum tunneling current for the $260 \mathrm{pA}$ tip. From the fabrication of TEM lamellae, it is known that the structuring by high ion currents causes splitting and amorphization of the nanostructure. For this reason, TEM lamellae are cleaned in the final step by an ion current as low as possible. The same mechanism can be assumed for the production of FIB structures by deposition. However, in the case of FIBID tips, this supports the suitability for applying tunneling electrodes since the splitting by an increased ion current leads to denser distribution of smaller Pt grains. In summary, it can be seen that the combination of a sufficient amount of $\mathrm{Pt}$, small grains, and dense distribution of the grains in the nanostructure is necessary to perform tunneling applications with FIBID/FEBID metal-organic tips.

\section{Conclusions/Summary}

The present study compared several PtC tips by FIBID and FEBID using HRTEM analysis. The differences in the tips can be specifically seen in the location, size, and distribution of the Pt grains. In particular, the FEBID tips show small grains and a greater amount of DLC, as evidenced by the spacing between the grains. The difference in the FIBID tips can also be seen in the size of the grains as the ion current varies. Due to the reduction in grain size with larger ion currents, they are more densely distributed and have less spacing between them. In particular, the FFT analysis of the FIBID and FEBID tips shows a change in the intensity of the outer diffraction rings corresponding to the 220 and 311 planes. The comparison of the FEBID tip and 260 pA FIBID tip proves the correlation between ring intensity and the frequency of smaller grains. For the FIBID tips of lower currents, particularly the $9 \mathrm{pA}$ tip, the outer rings are only faintly visible. The reason for this is the larger Pt grains, which can be detected by image analysis and the dark area in the microstructure. Smaller and more homogeneously distributed grains in the microstructure increase the probability of their occurrence in the edge region and, thus, specifically a higher suitability of the tunneling effect when two tips approach each other. This is shown by comparing the measurements of the tips integrated into a MEMS. The approach of the $9 \mathrm{pA}$ tips leads to ohmic contact. The tunneling effect does not occur. In contrast, the $260 \mathrm{pA}$ tips show a reproducible exponential increase and decrease of the current course corresponding to the tunneling effect. Therefore, a clear correlation can be shown between beam technology, grain size, and distribution in the nanostructure, as well as the percentage of $\mathrm{Pt}$ in the carbon matrix, and suitability for use with the tunneling effect.

Author Contributions: Conceptualization, M.H. and T.G.; methodology, M.H. and T.G.; validation, M.H., M.B. and T.G.; formal analysis, M.H. and M.B.; investigation, M.H.; data curation, M.H.; writing—original draft preparation, M.H.; writing—review and editing, M.H., M.B., T.G. and A.Z.; visualization, M.H.; supervision, A.Z. and T.G.; funding acquisition, A.Z. All authors have read and agreed to the published version of the manuscript.

Funding: This research was funded by Deutsche Forschungsgemeinschaft (DFG), grant number 321474168 and 386746281.

Institutional Review Board Statement: Not applicable.

Informed Consent Statement: Not applicable.

Data Availability Statement: All data used are shown in the text. Raw data are available on request.

Acknowledgments: The authors acknowledge the funding of the Deutsche Forschungsgemeinschaft, which made this work possible. Furthermore, the authors thank Ernst Müller for his great technical and advisory support in bonding the chips.

Conflicts of Interest: The authors declare no conflict of interest. 


\section{References}

1. Matsui, S.; Kaito, T.; Fujita, J.-I.; Komuro, M.; Kanda, K.; Haruyama, Y. Three-dimensional nanostructure fabrication by focusedion-beam chemical vapor deposition. J. Vac. Sci. Technol. B Microelectron. Nanometer Struct. 2000, 18, 3181-3184. [CrossRef]

2. Allameh, S.; Yao, N.; Soboyejo, W. Synthesis of self-assembled nanoscale structures by focused ion-beam induced deposition. Scr. Mater. 2004, 50, 915-919. [CrossRef]

3. Reyntjens, S.; Puers, R. Focused ion beam induced deposition: Fabrication of three-dimensional microstructures and Young's modulus of the deposited material. J. Micromech. Microeng. 2000, 10, 181-188. [CrossRef]

4. Li, W.; Warburton, P.A. Low-current focused-ion-beam induced deposition of three-dimensional tungsten nanoscale conductors. Nanotechnology 2007, 18, 485305. [CrossRef]

5. Lin, J.-F.; Bird, J.P.; Rotkina, L.; Bennett, P.A. Classical and quantum transport in focused-ion-beam-deposited Pt nanointerconnects. Appl. Phys. Lett. 2003, 82, 802-804. [CrossRef]

6. Sadki, E.H.; Ooi, S.; Hirata, K. Focused-ion-beam-induced deposition of superconducting nanowires. Appl. Phys. Lett. 2004, 85, 6206-6208. [CrossRef]

7. De Marzi, G.; Iacopino, D.; Quinn, A.J.; Redmond, G. Probing intrinsic transport properties of single metal nanowires: Direct-write contact formation using a focused ion beam. J. Appl. Phys. 2004, 96, 3458-3462. [CrossRef]

8. Hernández-Ramírez, F.; Rodríguez, J.; Casals, O.; Russinyol, E.; Vilà, A.; Romano-Rodríguez, A.; Morante, J.; Abid, M. Characterization of metal-oxide nanosensors fabricated with focused ion beam (FIB). Sens. Actuators B Chem. 2006, 118, 198-203. [CrossRef]

9. Tham, D.; Nam, C.-Y.; Fischer, J.E. Microstructure and Composition of Focused-Ion-Beam-Deposited Pt Contacts to GaN Nanowires. Adv. Mater. 2005, 18, 290-294. [CrossRef]

10. Vilà, A.; Hernández-Ramírez, F.; Rodriguez, J.; Casals, O.; Romano-Rodriguez, A.; Morante, J.; Abid, M. Fabrication of metallic contacts to nanometre-sized materials using a focused ion beam (FIB). Mater. Sci. Eng. C 2006, 26, 1063-1066. [CrossRef]

11. Tao, T. Focused ion beam induced deposition of platinum for repair processes. J. Vac. Sci. Technol. B Microelectron. Nanometer Struct. 1991, 9, 162. [CrossRef]

12. Wägner, A. X-ray mask repair with focused ion beams. J. Vac. Sci. Technol. B Microelectron. Nanometer Struct. 1990, 8, 1557. [CrossRef]

13. De Marco, A.J.; Melngailis, J. Maskless fabrication of JFETs via focused ion beams. Solid-State Electron. 2004, 48, 1833-1836. [CrossRef]

14. Gazzadi, G.C.; Frabboni, S. Fabrication of $5 \mathrm{~nm}$ gap pillar electrodes by electron-beam Pt deposition. J. Vac. Sci. Technol. B Microelectron. Nanometer Struct. 2005, 23, L1. [CrossRef]

15. Haub, M.; Bogner, M.; Guenther, T.; Zimmermann, A.; Sandmaier, H. Development and Proof of Concept of a Miniaturized MEMS Quantum Tunneling Accelerometer Based on PtC Tips by Focused Ion Beam 3D Nano-Patterning. Sensors 2021, $21,3795$. [CrossRef]

16. Penate-Quesada, L.; Mitra, J.; Dawson, P. Non-linear electronic transport in Pt nanowires deposited by focused ion beam. Nanotechnol. 2007, 18, 215203. [CrossRef]

17. Ballestar, A.; Esquinazi, P. Transport characteristics of focused beam deposited nanostructures. Nanofabrication 2015, 2. [CrossRef]

18. Fransson, J.; Lin, J.-F.; Rotkina, L.; Bird, J.P.; Bennett, P.A. Signatures of bandlike tunneling in granular nanowires. Phys. Rev. B 2005, 72, 113411. [CrossRef]

19. Fernández-Pacheco, A.; De Teresa, J.M.; Córdoba, R.; Ibarra, M.R. Metal-insulator transition in Pt-C nanowires grown by focused-ion-beam-induced deposition. Phys. Rev. B 2009, 79, 174204. [CrossRef]

20. Rotkina, L.; Lin, J.-F.; Bird, J.P. Nonlinear current-voltage characteristics of Pt nanowires and nanowire transistors fabricated by electron-beam deposition. Appl. Phys. Lett. 2003, 83, 4426. [CrossRef]

21. Labille, J.; Pelinovskaya, N.; Botta, C.; Bottero, J.-Y.; Masion, A.; Joag, D.S.; Forbes, R.G.; Burger, S.; Pomplun, J.; Schmidt, F; i et al. Focused-Ion-Beam Chemical-Vapor-Deposition (FIB-CVD). In Encyclopedia of Nanotechnology; Springer: Dordrecht, The Netherlands, 2012; pp. 866-876.

22. Kometani, R.; Ichihashi, T.; Kanda, K.; Suzuki, T.; Niihara, K.; Ishihara, S.; Kaito, T.; Matsui, S. Resistivity change of the diamondlike carbon, deposited by focused-ion-beam chemical vapor deposition, induced by the annealing treatment. J. Vac. Sci. Technol. B Microelectron. Nanom. Struct. 2008, 26, 2628-2631. [CrossRef]

23. De Teresa, J.M.; Córdoba, R.; Fernández-Pacheco, A.; Montero, O.; Štrichovanec, P.; Ibarra, M.R. Origin of the Difference in the Resistivity of As-Grown Focused-Ion- and Focused-Electron-Beam-Induced Pt Nanodeposits. J. Nanomater. 2009, $2009,936863$. [CrossRef]

24. Koops, H.W.P. Fabrication and characterization of platinum nanocrystalline material grown by electron-beam induced deposition. J. Vac. Sci. Technol. B Microelectron. Nanometer Struct. 1995, 13, 2400. [CrossRef]

25. Van Dorp, W.F.; Hagen, C.W. A critical literature review of focused electron beam induced deposition. J. Appl. Phys. 2008, 104, 081301. [CrossRef]

26. Utke, I.; Hoffmann, P.; Melngailis, J. Gas-assisted focused electron beam and ion beam processing and fabrication. J. Vac. Sci. Technol. B Microelectron. Nanom. Struct. 2008, 26, 1197. [CrossRef]

27. Wiedemair, J.; Menegazzo, N.; Pikarsky, J.; Booksh, K.S.; Mizaikoff, B.; Kranz, C. Novel electrode materials based on ion beam induced deposition of platinum carbon composites. Electrochimica Acta 2010, 55, 5725-5732. [CrossRef] 
28. Langford, R.M.; Wang, T.-X.; Ozkaya, D. Reducing the resistivity of electron and ion beam assisted deposited Pt. Microelectron. Eng. 2007, 84, 784-788. [CrossRef]

29. Lin, J.-F.; Bird, J.P.; Rotkina, L.; Sergeev, A.; Mitin, V. Large effects due to electron-phonon-impurity interference in the resistivity of Pt/C-Ga composite nanowires. Appl. Phys. Lett. 2004, 84, 3828. [CrossRef]

30. An, B.-S.; Kwon, Y.; Oh, J.-S.; Shin, Y.-J.; Ju, J.-S.; Yang, C.-W. Evaluation of ion/electron beam induced deposition for electrical connection using a modern focused ion beam system. Appl. Microsc. 2019, 49, 1-5. [CrossRef]

31. Tao, T. Focused ion beam induced deposition of platinum. J. Vac. Sci. Technol. B Microelectron. Nanometer Struct. 1990, 8, 1826. [CrossRef]

32. Melngailis, J. Focused Ion Beam Induced Deposition: A Review. In Proceedings of the Proc. SPIE 1465, Electron-Beam, X-Ray, and Ion-Beam Submicrometer Lithographies for Manufacturing; Peckerar, M.C., Ed.; SPIE: San Jose, CA, USA, 1 August $1991 ;$ p. 36.

33. Schwalb, C.H.; Grimm, C.; Baranowski, M.; Sachser, R.; Porrati, F.; Reith, H.; Das, P.; Müller, J.; Völklein, F.; Kaya, A.; et al. A Tunable Strain Sensor Using Nanogranular Metals. Sensors 2010, 10, 9847-9856. [CrossRef] [PubMed]

34. Gates-Rector, S.; Blanton, T. The Powder Diffraction File: A quality materials characterization database. Powder Diffr. 2019, 34, 352-360. [CrossRef] 